\title{
IDENTIFYING THE KEY ENVIRONMENTAL FACTORS AND BACTERIAL COMMUNITIES IN HUMIFICATION AND THEIR RELATIONSHIPS DURING GREEN WASTE COMPOSTING
}

\author{
LI, L. ${ }^{1}-$ GUO, X. P. ${ }^{1 *}-$ ZHAO, T. N. ${ }^{1}-$ LIU, L. ${ }^{2}-$ LI, T. Y. ${ }^{3}$ \\ ${ }^{I}$ College of Soil and Water Conservation, Beijing Forestry University, No.35 Tsinghua East \\ Road, Beijing 100083, PR China \\ ${ }^{2}$ Xiong'an Institute of Eco-Environment, Hebei University, No. 180 Wusi East Road, Baoding, \\ 071002 Hebei Province, PR China \\ ${ }^{3}$ Tsinghua Tongheng Urban Planning \& Design Institute, Qinghe Middle Street, Beijing \\ 100085, PR China \\ *Corresponding author \\ e-mail: guoxp@bjfu.edu.cn
}

(Received $23^{\text {rd }}$ Mar 2020; accepted $6^{\text {th }}$ Oct 2020)

\begin{abstract}
In this study, the key factors affecting the formation of humic substances (HS) during green waste composting (GWC) and their potential relationships were investigated by setting up different initial particle sizes (IPS) (2 and $5 \mathrm{~mm}$ ) and $\mathrm{C} / \mathrm{N}$ (23, 30, and 37) for composting, evaluating the dynamic of environmental factors and humification parameters during the composting, and performing high-throughput sequencing to detect the bacterial structure and diversity dynamics. The results showed that the $2 \mathrm{~mm}$ and $\mathrm{C} / \mathrm{N}=23$ treatment could promote the degradation of organic matter, increase the fermentation temperature and germination index (GI), and generate a high HS content and humification degree, indicating that the IPS and $\mathrm{C} / \mathrm{N}$ ratio were the potential controlling factors for composting maturation. Taxonomic analysis showed that the bacterial community structure was significantly different at various stages of composting, while the bacterial community composition of the same composting stage for the different treatments was similar. In addition, a correlation analysis and redundancy analysis showed that the formation of HS was significantly affected by temperature, GI, polyphenols and polysaccharides. Anaerolineales, Cytophagales and Blastocatellales were the important microbial markers of the maturation process. The above results provide insights and potential methods for regulating HS formation during the composting process.
\end{abstract}

Keywords: compost, humic substances, precursor, maturity, high-throughput sequencing, redundancy analysis

\section{Introduction}

With the development of ecological construction, the quantity of green waste (GW) could increase accordingly. Because $\mathrm{GW}$ is rich in organic matter and abundant nutrient elements, composting treatments represent an effective method of recycling GW. Composting is an aerobic process through which organic matter is decomposed into HS under a characteristic microbial succession. The composting environment and composition and succession of microbial communities all have an impact on the formation of HS (Guo et al., 2019); therefore, we investigated the multidirectional feedback relationship between HS, environmental parameters and microbial communities to determine the key factors that affect the formation of HS. This study provides insights into the regulation of HS formation to improve the quality of GWC.

HS is an important product of aerobic fermentation of organic wastes and an important evaluation standard for the quality of fermented products. During the 
composting process, the formation of HS is extremely complex and affected by environmental factors, such as temperature, $\mathrm{C} / \mathrm{N}$ ratio and particle size, and differences are observed in the influences of different composting processes. For example, studies have shown that composting could be effectively performed at low $\mathrm{C} / \mathrm{N}$ (Huang et al., 2004; Zhu, 2007), while Silva et al. (2014) found that a low initial C/N could impede the stability of HS and affect the quality of composting. Both Zhang and Sun (2014) and Liu et al. (2018) believed that the appropriate IPS was a potential controlling factor for the physicochemical properties of composting and bacterial diversity, which could accelerate the composting process, although no unified standard was proposed. In addition, recent studies have demonstrated that the precursors of HS, such as polyphenols, proteins, and polysaccharides, have significant effects on the formation of HS (Wu et al., 2017); and Zhang et al. (2018) believed that the addition of exogenous precursors could also regulate the formation of HS. Considering the differences in the element composition and properties of different raw materials, the effect of environmental factors on the rate of HS synthesis and quality of HS varies greatly during the composting process (Wang et al., 2015). Therefore, the relationship between environmental factors and HS in GWC needs further investigation.

Microbial activities have a vital impact on the formation of HS (Song et al., 2014). Studies have shown that microbial diversity interacts with organic matter transformation and HS stability during the composting process (Xi et al., 2016; Zhang et al., 2016), during which bacteria have a strong and considerable influence due to their high quantity and good adaptability to adverse environmental conditions (López-González et al., 2015; Antunes et al., 2016). Bacteria have different activities during different composting stages, which may lead to different formation patterns of HS during the composting process. Therefore, it is important to study the community composition and dynamics of bacteria during the composting process to understand the overall mechanisms underlying the composting humification process. In addition, considering that complex composting environmental factors could influence the composition of microbial communities (Wang et al., 2015; Tortosa et al., 2017), the interactions between environmental factors, bacterial communities and HS must be clarified. Wu et al. (2017) studied the bacterial communities that affect HS using the traditional denaturing gradient gel electrophoresis (DGGE) technique to assess bacterial communities; however, this method is limited by the taxonomic resolution and cannot fully clarify the structure and diversity of composting communities. Therefore, detailed information on the bacterial community dynamics during humification is still lacking. In this study, the high-throughput sequencing method was used to comprehensively and accurately reflect the microbial community structure and objectively reflect the low-abundance but important functional microbes to provide thorough and accurate microbial information to explore the bacterial community succession pattern during the GWC process and its effect on HS.

Therefore, the objectives of this study are to 1) investigate the dynamic changes in environmental factors and the evolution of bacterial community diversity during GWC under different $\mathrm{C} / \mathrm{N}$ ratios and IPS and 2) clarify the relationships among environmental factors, bacterial communities and HS during the GWC process. This study aimed to identify the key factors that affect HS formation during GWC and promote the formation of HS through the regulation of key influencing factors to provide new insights and technical references for improving the quality of GWC. 


\section{Materials and methods}

\section{Composting materials}

GW is supplied by Shoufa Tianren Co. Ltd. in Beijing, China, and the degree of lignification is high. Based on studies by Hanc and Dreslova (2016) and Liu et al. (2018), the waste materials were crushed to two sizes of $2 \mathrm{~mm}$ and $5 \mathrm{~mm}$ for composting. Urea and sawdust were purchased from Kaiyin Horticulture Co., Ltd. and used to adjust the initial $\mathrm{C} / \mathrm{N}$ of composting. Combining the results of previous studies (Huang et al., 2004; Silva et al., 2014) and preliminary experiments, three C/N ratios of 23, 30, and 37 were set to investigate the effect of the $\mathrm{C} / \mathrm{N}$ ratio for GWC (Table 1). The microbial inoculum was purchased from Jingpuyuan Co., Ltd. to accelerate the initial composting (Zhang and Sun, 2014). Table 2 lists basic properties of the green waste and initial composts.

Table 1. Set parameters of particle size and $C / N$

\begin{tabular}{c|c|c}
\hline Treatment & Particle size $(\mathbf{m m})$ & $\mathbf{C} / \mathbf{N}$ \\
\hline T1 & 2 & 23 \\
T2 & 2 & 30 \\
T3 & 2 & 37 \\
T4 & 5 & 23 \\
T5 & 5 & 30 \\
T6 & 5 & 37 \\
\hline
\end{tabular}

Table 2. Basic properties of green waste and initial composts

\begin{tabular}{c|c|c|c|c|c}
\hline Treatment & TOC\% & TN\% & C/N ratio & pH value & EC $(\mathbf{m S / c m})$ \\
\hline GW & $34.54 \pm 0.95$ & $1.20 \pm 0.05$ & $28.76 \pm 0.24$ & $6.86 \pm 0.03$ & $0.97 \pm 0.12$ \\
T1 & $33.31 \pm 1.62$ & $1.39 \pm 0.06$ & $23.90 \pm 0.17$ & $7.25 \pm 0.07$ & $1.19 \pm 0.08$ \\
T2 & $34.01 \pm 1.37$ & $1.12 \pm 0.04$ & $30.43 \pm 0.68$ & $7.15 \pm 0.05$ & $0.92 \pm 0.04$ \\
T3 & $37.22 \pm 0.65$ & $1.02 \pm 0.04$ & $36.50 \pm 0.77$ & $7.05 \pm 0.05$ & $0.86 \pm 0.00$ \\
T4 & $34.49 \pm 2.40$ & $1.45 \pm 0.08$ & $23.76 \pm 1.00$ & $7.40 \pm 0.08$ & $1.00 \pm 0.03$ \\
T5 & $35.01 \pm 0.48$ & $1.14 \pm 0.08$ & $30.71 \pm 0.38$ & $7.00 \pm 0.03$ & $0.82 \pm 0.06$ \\
T6 & $36.10 \pm 1.21$ & $0.96 \pm 0.07$ & $37.41 \pm 0.73$ & $7.00 \pm 0.06$ & $0.79 \pm 0.04$ \\
\hline
\end{tabular}

Notes: Data are reported as mean $(n=3)$. TOC: total organic carbon; TN: total nitrogen; EC: electrical conductivity

\section{Composting procedure and sampling}

A total of 6 treatments were performed, with 3 replicates for each treatment (Table 1). The mixture was placed into plastic reactors (Fig. 1) with a diameter of $0.25 \mathrm{~m}$, a height of $0.31 \mathrm{~m}$ and a volume of $10 \mathrm{~L}$. A number of ventilation holes were left at the top, and a thermometer was fixed in the middle of the reactors to monitor the temperature change during the process. The reactor was placed in an incubator to compensate for the heat loss and simulate the self-heating process during the composting process. The temperature of the climate chamber was kept below the temperature of the fermenter by $3 \sim 5{ }^{\circ} \mathrm{C}$ ( $\mathrm{Li}$ et al., 2013), and the composting process lasted 30 days. The water content in the compost was adjusted to $65 \%$ by the addition of 
distilled water, and the water content was maintained at 55\%-65\% during the composting process. The reactors were manually flipped for 2 minutes every 2 days to ensure that sufficient oxygen was available. On the $0,2,5,9,15,22$ and 30 days, $150 \mathrm{~g}$ (wet weight) of the sample from each fermenter was collected, and it was divided into two parts: one part was air dried, the other part was stored at $4{ }^{\circ} \mathrm{C}$. On the 2,9 , and 30 days, the samples were immediately subjected to microbiological examination. The samples used for the microbial analysis were labelled as T1D2-T6D2, T1D9-T6D9 and T1D30-T6D30, and they represented the composting heating phase, thermophilic phase and maturity phase.

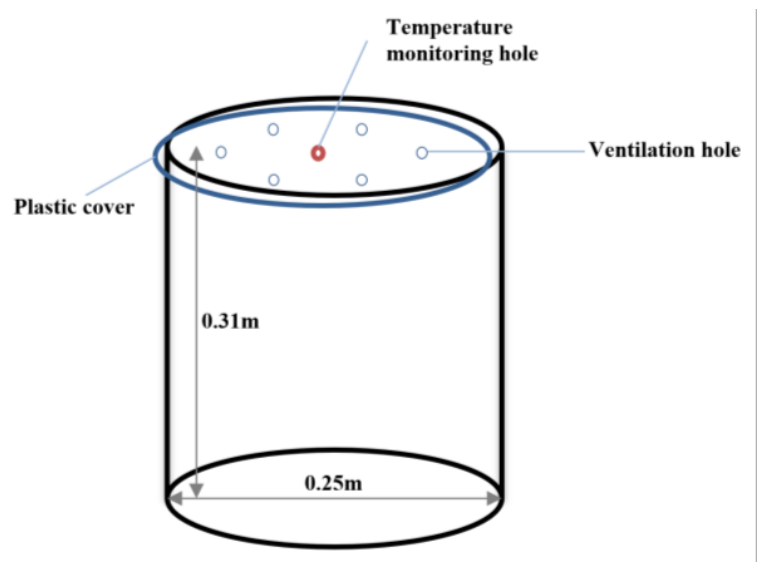

Figure 1. The illustration of the composting reactor

\section{Chemical and physical analysis}

Air-dried samples were used to determine the total organic carbon (TOC), total nitrogen (TN), humic substance (HS), humic acids (HA), fulvic acid (FA), as described by Liu et al. (2018). Water content was measured with oven drying method (Zhang et al., 2020). The $\mathrm{E}_{4} / \mathrm{E}_{6}$ ratio is the absorbance ratio at wavelengths 465 and $665 \mathrm{~nm}$, which was measured using an ultraviolet spectrophotometer (TU-1810DS). The humification indexes were calculated as $\mathrm{Wu}$ et al. (2017) described. The $\mathrm{pH}$, electrical conductivity (EC), germination index (GI) were measured using fresh samples in accordance with Li et al. (2012), GR (germination rate) and GI was calculated as follows (Zhang and Sun, 2019):

$$
\begin{gathered}
F_{\mathrm{GR}}=\frac{S_{\mathrm{T}}}{S_{\mathrm{C}}} \times 100 \% \\
F_{\mathrm{GI}}=\frac{S_{\mathrm{T}} \cdot L_{\mathrm{T}}}{S_{\mathrm{C}} \cdot L_{\mathrm{C}}} \times 100 \%
\end{gathered}
$$

where $F_{\mathrm{GR}}-$ germination rate; $S_{\mathrm{T}}-$ seed germination of treatment; $S_{\mathrm{C}}-$ seed germination of control; $F_{\mathrm{GI}}-$ germination index; $L_{\mathrm{T}}$ - average root length of treatment $(\mathrm{mm}) ; L_{\mathrm{C}}$ - average root length of control ( $\left.\mathrm{mm}\right)$.

The amino acid content was determined using a ninhydrin color liquid. The total polysaccharide and reducing sugar contents were determined using an anthrone reagent 
and dinitrosalicylic acid (DNS) reagent. The calcium acetate method reported by Zhang et al. (2018) was used to determine the carboxyl group content. For detection of polyphenolic compounds contents, composting samples were extracted with $80 \%$ methanol, and the extraction was analysed by the Folin-Ciocalteu assay using an ultraviolet spectrophotometer (Li et al., 2015).

\section{Microbial analysis}

The DNA was extracted from fresh compost samples using the E.Z.N.A. ${ }^{\circledR}$ Bacterial DNA Kit (Omega Bio-tek, Norcross, GA, USA) following the manufacturer's protocol. Agarose gel electrophoresis $(1 \%)$ and spectrophotometry were applied to detect the purity and quantity of the extracted DNA. Using universal primers forward $338 \mathrm{~F}$ (5'-ACTCCTACGGGAGGCAGCAG-3') and the reverse 806R (5'-GACTACHVGGGTWTCTAAT-3') amplified the bacterial 16S rRNA gene (V3-V4 hypervariable region). Polymerase chain reaction (PCR) condition and the procedure were performed as Liu et al. (2018). Moreover, the PCR amplicons were extracted and purified using agarose gels (2\%) and AxyPrep DNA Gel purification kit (AXYGEN Inc.). High-throughput sequencing and sequence read archive were on the Illumina MiSeq PE300 sequencing platform (Illumina Inc., CA, USA) by Beijing Allwegene Tech Ltd. (Beijing, China). The raw sequence data were processed and analyzed in the QIIME 1.8.0 to obtain high-quality sequences.

\section{Statistical analysis}

All analyses were repeated three times. Dynamics of environmental parameters were assessed by ANOVAs at significance $\mathrm{p}<0.05$ level, when ANOVAs were significant, means were separated with an LSD test. For statistical analysis of data, Origin 2018 and SPSS version 23 were used. Besides, Canoco 5.0 was used to analyse the relationship among environmental factors, bacterial community composition, and humic substance by redundancy analysis.

\section{Results and discussion}

\section{Dynamics of environmental factors during composting}

\section{Temperature}

During the aerobic fermentation process, the oxidation reaction of microbes causes the self-heating effect of the compost, and the change in temperature reflects the severity of the decomposition activities of microorganisms (Dzulkurnain et al., 2017). As shown in the Fig. $2 a$, because of a suitable temperature and humidity create favorable conditions for fermentation, the temperature increased rapidly at the initial stage of composting, the average temperature at $5 \mathrm{~mm}\left(41.87^{\circ} \mathrm{C}\right)$ in the heating phase of the composting was higher than that of $2 \mathrm{~mm}\left(40.96^{\circ} \mathrm{C}\right)$, indicating that the $5 \mathrm{~mm}$ treatment had a stronger mineralization capacity, which might be due to the low initial oxygen content and lower microbial activity in the early stage caused by the small IPS. Afterwards, the proper compost turning treatment optimized the porosity of composting, the relatively high specific surface area of $2 \mathrm{~mm}$ IPS was more conducive to microbial growth and promoted heat accumulation. Thus, from heating phase to thermophilic phase, a larger average temperature increase was observed in the $2 \mathrm{~mm}$ treatments 


$$
-50 \text { - }
$$

$\left(10.59^{\circ} \mathrm{C}\right)$ than that of $5 \mathrm{~mm}\left(8.61^{\circ} \mathrm{C}\right)$. During the whole composting process, the order of the average temperatures for the six treatments was $\mathrm{T} 1>\mathrm{T} 2>\mathrm{T} 4>\mathrm{T} 3>\mathrm{T} 5>\mathrm{T} 6$, the average temperature of the $2 \mathrm{~mm}$ IPS was higher than that of the $5 \mathrm{~mm}$ IPS and the average temperature decreased with increases in the $\mathrm{C} / \mathrm{N}$ ratio.
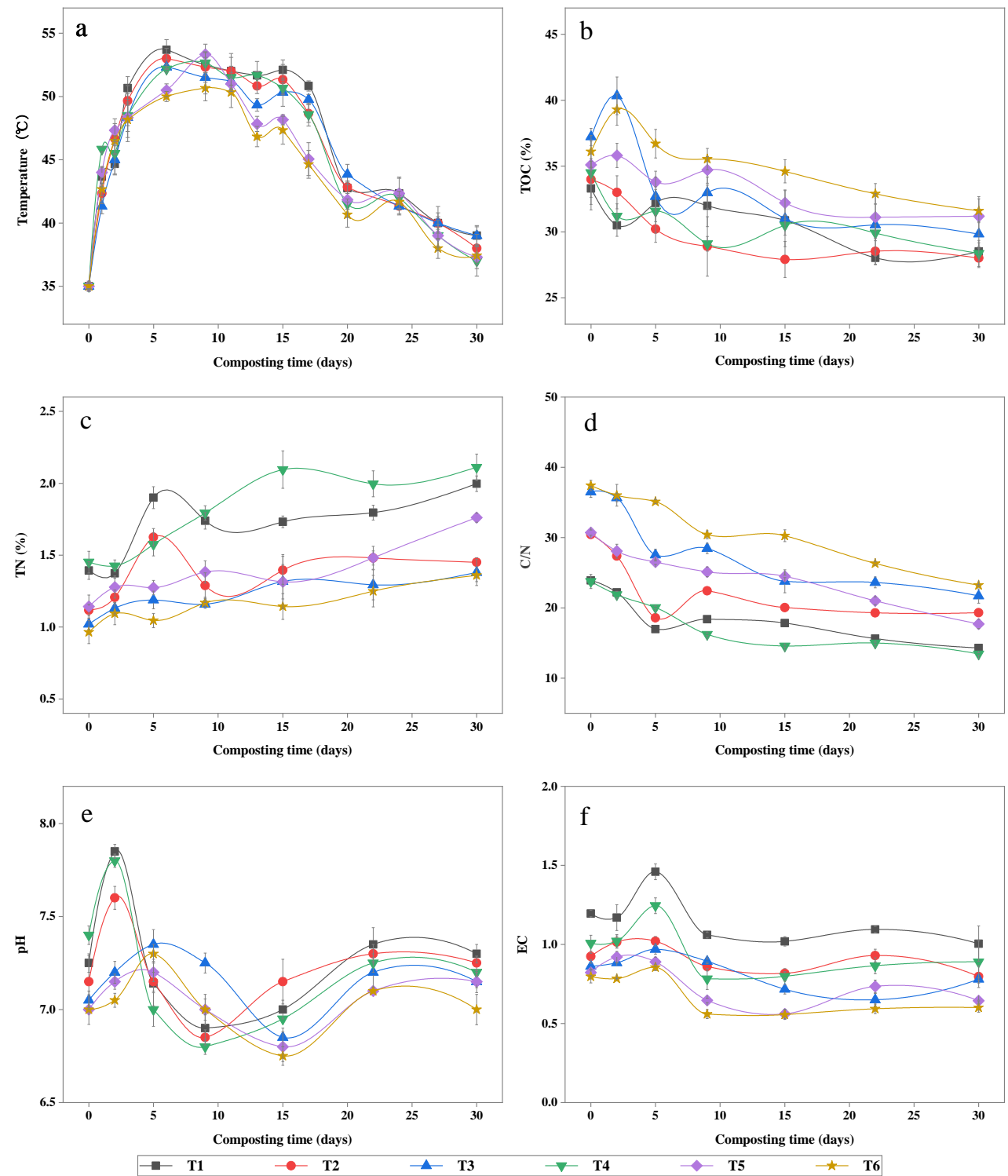

Figure 2. Dynamic of temperature (a), TOC (b), TN(c), C/N (d), pH (e) and EC (f) during composting [Error bars represent standard deviation (means $\pm S D, n=3$ )]

\section{C/N ratio}

As shown in Fig. 2b,c, compared with the initial composting, the organic carbon content of all composting products decreased and the TN content increased. The average reduction of organic carbon in the $2 \mathrm{~mm}$ treatment $(6.04 \%)$ was greater than those of the $5 \mathrm{~mm}$ treatment (4.84\%), indicating that the $2 \mathrm{~mm}$ IPS of GWC was more conducive to the decomposition activities of microorganisms. 
The $\mathrm{C} / \mathrm{N}$ ratio reflects the progress of mineralization in the composting process and is closely related to the maturity of the composting product (Khalil et al., 2008; Lobna et al., 2019). In this study, the organic carbon in the compost was catabolized faster than nitrogen under microbial action, all treatments $\mathrm{C} / \mathrm{N}$ ratio showed a decreasing trend (Fig. 2d). Among them, the final $\mathrm{C} / \mathrm{N}$ of $\mathrm{T} 1$ and $\mathrm{T} 4$ treatments is significantly lower than other treatments $(\mathrm{p}<0.05)$, which indicating that the low initial $\mathrm{C} / \mathrm{N}$ ratio can accelerate the mineralization process of composting, thereby effectively promoting humification and improving the maturity of composting products.

\section{pH and EC}

$\mathrm{pH}$ plays an important role in the activity of composting microorganisms. Researchers in the composting field believe that the activity and reproductive capacity of common microorganisms are highest when the $\mathrm{pH}$ value is 6.5-7.5 (Zhang and Sun, 2018). In this study, the $\mathrm{pH}$ first increased, then decreased, and then fluctuated to a steady state (Fig. $2 e$ ). The $\mathrm{pH}$ in the $2 \mathrm{~mm}$ treatments started to increase faster than that in the $5 \mathrm{~mm}$ treatments. In the same IPS treatments, the lower the $\mathrm{C} / \mathrm{N}$ ratio, the higher the $\mathrm{pH}$ value, which is because the increase in microbial activity at the initial stage could increase the decomposition of easily degradable organic nitrogen, thereby increasing the release of ammonia and resulting in a sharp increase in $\mathrm{pH}$ at the initial stage. The low-C/N ratio treatments can provide more nitrogen sources for the microorganisms so that the $\mathrm{pH}$ increases even faster. The decrease in $\mathrm{pH}$ value in the late stage might be due to the release of $\mathrm{NH}_{3}$ and $\mathrm{H}^{+}$during the nitrification process (Wang et al., 2017).

EC reflects the total salt content in the compost (Awasthi et al., 2014) and thus can be used as an index for the decomposition and polymerization kinetics of organic matter. As shown as Fig. 2f, EC increased to a peak value in the early stage of decomposition and then decreased and stabilized with the binding and conversion of soluble products (such as organic acids and salts) into HS (Petric et al., 2012; Chen et al., 2017). The $2 \mathrm{~mm}$ IPS treatments had a high EC value, indicating that these treatments contained more salts and small molecules, which may be related to the high specific surface area of the small IPS treatments, thus increasing the ion exchange capacity.

\section{Precursors}

Polyphenols, carboxyl, amino acids, reducing sugars and polysaccharides are considered to be involved in the formation of HS through biochemical synthesis (Guo et al., 2019). The Fig. 3a-e showed changes in 5 HS precursor concentrations during composting. The contents of amino acids and reducing sugars showed a decreasing trend, the contents of phenolic and carboxyl groups first increased and then decreased, and the total polysaccharides showed an overall upward trend. The trends of different treatments were basically consistent, and the concentration changed more dramatically during the heating and thermophilic phases because of the many easily degradable substances and high activity of microorganisms in the early stage of composting. Under intense microbial degradation, lignin is partially degraded to produce small molecule polyphenols, and simultaneously, abundant enzymes are secreted to degrade fat and oxidize hydroxyl groups to form carboxyl groups; thus, polyphenols and carboxyl concentration are increased in the early stage. The produced amino acids and sugars, which are the main energy sources of microorganisms, are used in large quantities, and their concentrations decrease. 

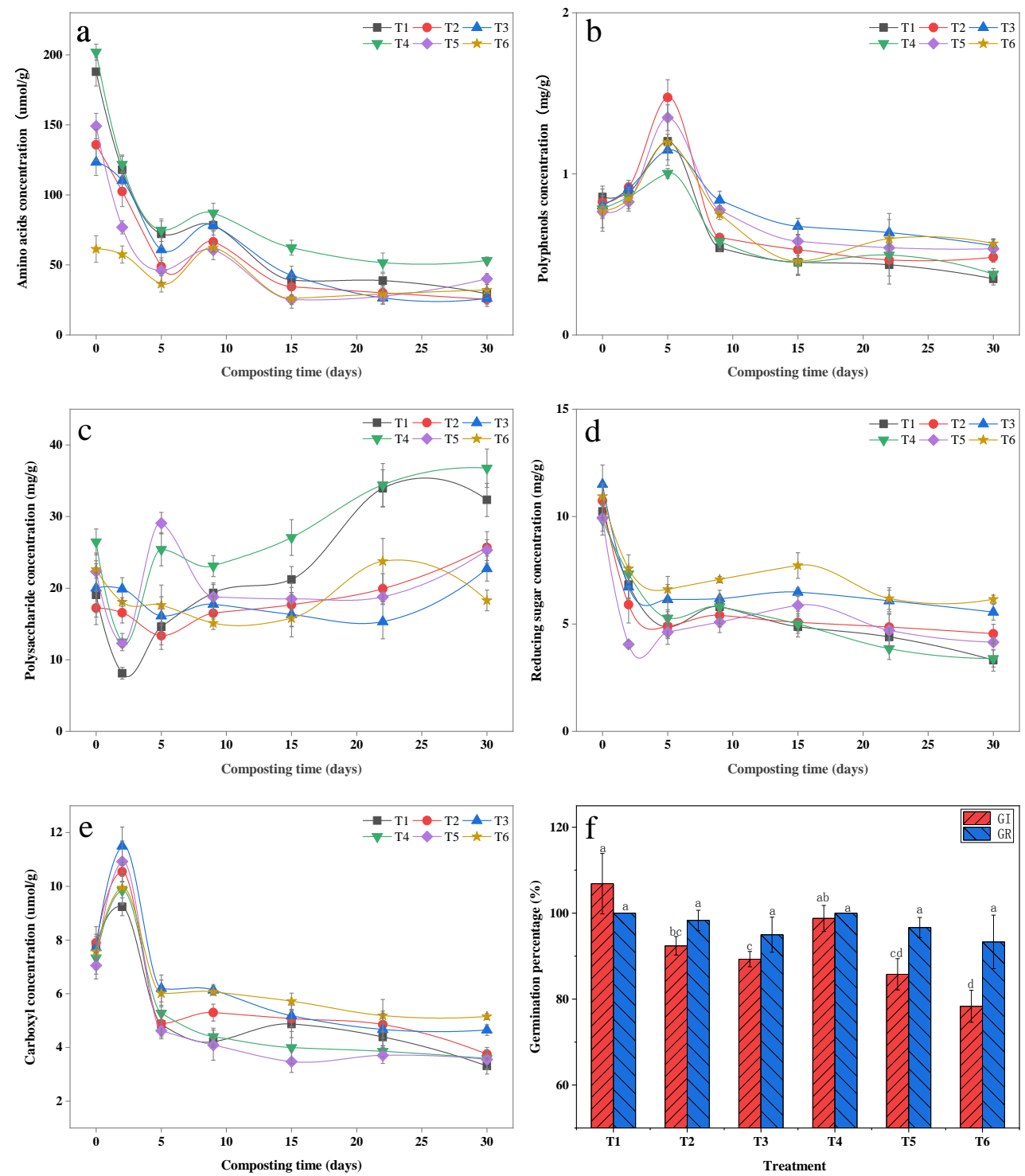

Figure 3. Changes in the precursors including(a) amino acids, (b) Polyphenols, (c) polysaccharides, $(d)$ reducing sugars, (e) carboxyl; and (f)GI in different composting treatments [Error bars represent standard deviation (means $\pm S D, n=3$ ); germination percentage with the same letter are not significantly different at $p<0.05]$

\section{Phytotoxicity of the compost products}

The germination index (GI) and germination rate (GR) can reflect the phytotoxicity and maturity of composting. High maturity can promote plant growth, whereas high levels of soluble salts, organic acids and $\mathrm{pH}$ can cause composting phytotoxicity (Wang et al., 2017). In this study, the GI gradually increased with composting time, and Fig. $3 f$ showed the final GI (calculated as Eq.1) was ranked as T1>T4>T2>T5>T3>T6, GR (calculated as Eq.2) was ranked as T1=T4>T2>T5>T3>T6. The results of two-way ANOVA showed that $\mathrm{C} / \mathrm{N}$ and IPS significantly affected GI $(\mathrm{p}<0.05)$, but the interaction between them was not significant. The $2 \mathrm{~mm}$ IPS and $\mathrm{C} / \mathrm{N}=23$ treatment can significantly improve the GI value, indicating that this treatment was more conducive to the degradation of toxic substances. GI value $>80 \%$ indicates that composting is 


$$
-53 \text { - }
$$

completely decomposed (Zucconi et al., 1981), and based on this threshold, T1-T5 are all at a mature level.

\section{Dynamic changes in $\mathrm{HS}$}

During the composting process, HS forms via $\mathrm{C}$ and $\mathrm{N}$ cycles. HA and FA are important components of HS and play a decisive role in the quality of HS to a large extent. At the initial stage of composting, the concentrations of HS and its components decrease overall (Fig. 4a-c), thus reflecting the degradation of unstable compounds in the structure (Barje et al., 2012). Because of the presence of many acid functional groups and the low molecular weight in the early stage, the water solubility of FA is higher than that of HA; thus, the concentration of FA is relatively high in the initial composting stage. Subsequently, a series of polycondensation reactions of the decomposed phenols, carbohydrates, and nitrogen-containing compounds result in an increase in the concentrations of HS and HA (Zhou et al., 2014), and the gradual decrease in FA suggesting that the readily available carbon in composting is reduced and the stability of the composting is increased. Two-way ANOVA showed that $\mathrm{C} / \mathrm{N}$ ratio had significant effect on $\mathrm{HS}(\mathrm{p}<0.05)$, the treatments with low $\mathrm{C} / \mathrm{N}$ ratio had higher HS content.

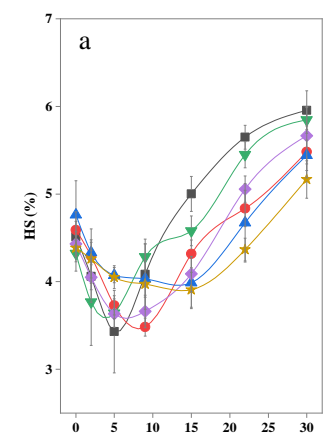

$\begin{array}{lllll}5 & 10 & 15 & 20 & 25 \\ & \text { Composting time (days) }\end{array}$
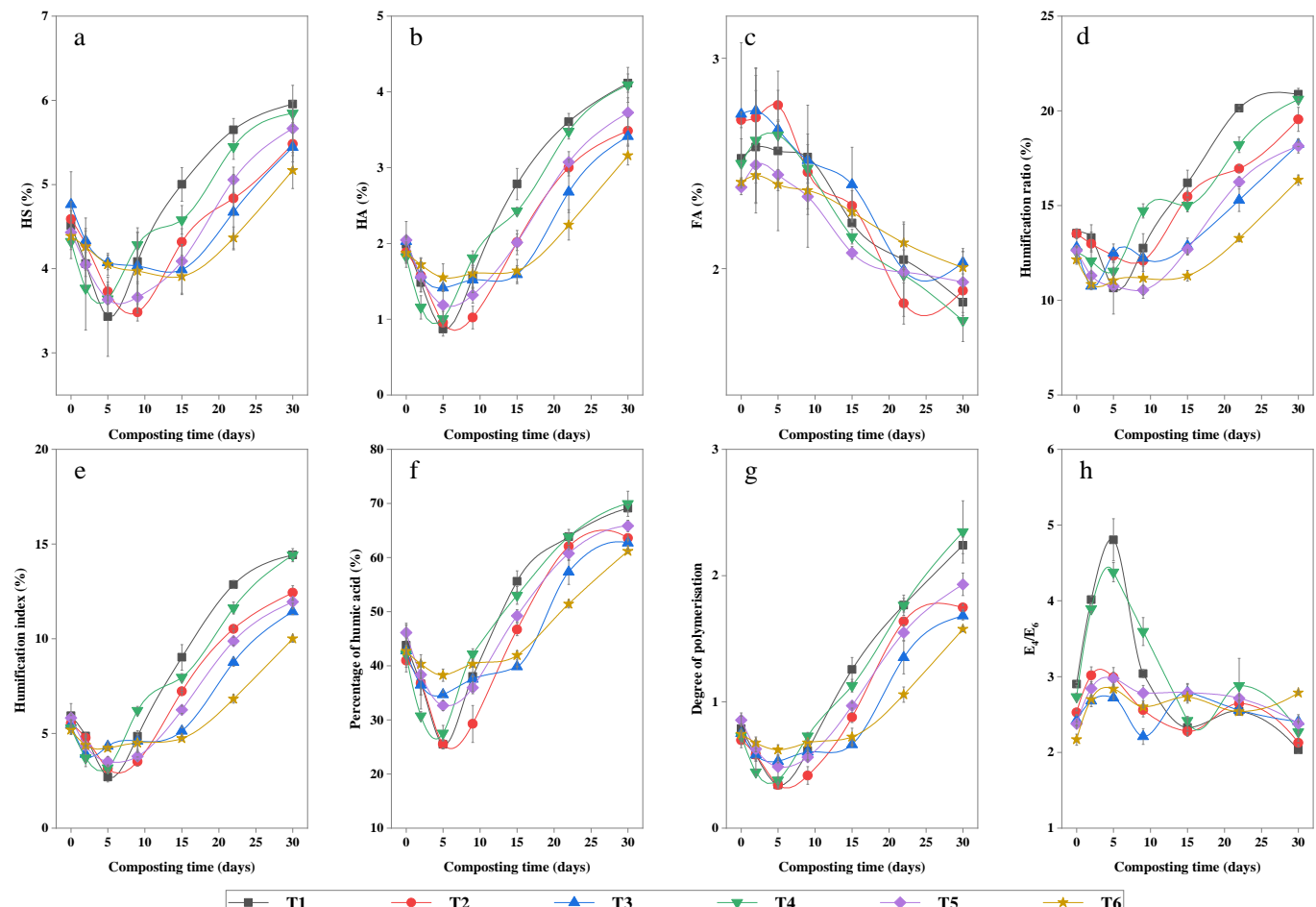

Figure 4. Variation in $H S(a), H A(b), F A(c), H I(d), H R(e), P H A(f), D P(g)$ and $E_{4} / E_{6}$ ratio( $(h)$ showing the humification process during the composting [Error bars represent standard deviation (means $\pm S D, n=3$ )]

In addition to the changes in the HS concentration, the humification-related indices (DP, HI, HR and PHA) are considered to more accurately reflect the evolution of humification (Sánchez-Monedero et al., 1999; Kulikowska and Klimiuk, 2011). These indices generally showed an upward trend (Fig. 4d-g), and the increases in HI and HR 
meant an increase in HS and a decrease in biodegradable organic matter, thus reflecting an increase in humification caused by composting. PHA and DP are the measurements of complex HA produced by FA, and an increase in these indices indicates an increase in the molecular weight of $\mathrm{HS}$ and structural complexity. $\mathrm{E}_{4} / \mathrm{E}_{6}$ is the ratio of the absorbance at $465 \mathrm{~nm}$ and $665 \mathrm{~nm}$, which can more intuitively describe the change in the polymerization degree of aromatic components in HS (Awasthi et al., 2014). $\mathrm{E}_{4} / \mathrm{E}_{6}$ fluctuated constantly during the composting process (Fig. 4h), indicating that decomposition and aggregation occurred throughout the entire composting process, and $\mathrm{E}_{4} / \mathrm{E}_{6}$ gradually decreased at the late stage, indicating the formation of more polycondensed HAs and a high degree of composting condensation.

\section{Dynamic changes in bacterial communities}

$16 \mathrm{~S}$ rDNA high-throughput sequencing technology is an important method for the rapid, flexible, efficient, and accurate acquisition of microbial community composition and diversity. A total of 1169664 high-quality bacterial sequences were obtained from the 16S rRNA clone library of GWC samples collected during different composting stages, and they aggregated into 2208 bacterial operational taxonomic units (OTUs) with a 3\% nucleotide difference. These 2208 OTUs belonged to 36 phyla. Proteobacteria, Chloroflexi, Firmicutes, Actinobacteria, Bacteroidetes, and Acidobacteria were the six most dominant phyla among the total treatments. The compost samples accounted for over $92 \%$ of the gene sequences. Previous studies also found these dominant bacteria in lignin waste composting and other types of composting (Awasthi, 2017a; Liu, 2018). The relative abundance of bacteria at the phylum level among all the treatments is shown in Fig. $5 a$.
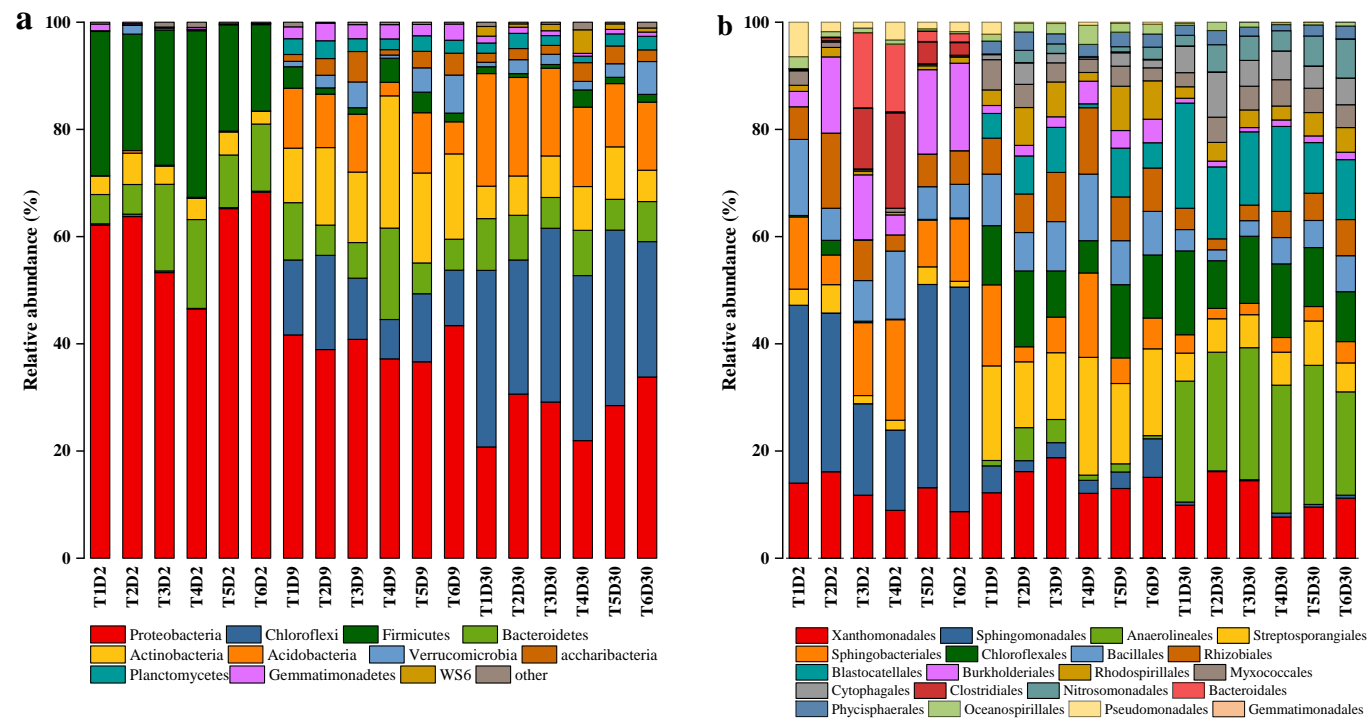

Figure 5. Relative abundance of the dominant bacteria in (a) phyla level and (b) order level by treatment

In the early stage of composting, which mainly focuses on the degradation of simple organic matter, Proteobacteria and Firmicutes are the most abundant and average abundances of $60 \%$ and $23 \%$, respectively. The abundance of Proteobacteria at the 
medium-C/N was the highest at $64.5 \%$; the abundance of Firmicutes at the low-C/N was the highest at 1.4 and 1.5 times that of the other two $\mathrm{C} / \mathrm{N}$ groups; and significant differences were not observed in the treatments with different particle sizes. The results suggested that the nutritional environment had a greater impact on bacterial activity at the initial stage of composting. Proteobacteria have been shown to play an important role in the degradation of small molecules (such as glucose, propionate, and butyrate) (Zhou et al., 2019). Because Firmicutes can effectively utilize carbohydrates, it is also the key for early composting (Ariesyady et al., 2007).

At the middle stage of composting, the mean abundance of Proteobacteria decreased to $40 \%$ and Actinobacteria increased from $3.9 \%$ to $15.9 \%$ to become the second major phylum, which was caused by Actinobacteria-induced microbial production of lignocellulosic enzymes. The lignocellulosic enzymes have an excellent effect on the degradation of lignocellulose (Taha et al., 2016), and their spores formed at high temperatures can resist the harsh environment during the composting process (growing at $50-60{ }^{\circ} \mathrm{C}$ ) (Zhao et al., 2016; Wei, 2018). The abundance of Actinobacteria in the $5 \mathrm{~mm}$ treatments was $55 \%$ higher than that in the $2 \mathrm{~mm}$ treatments, and the average abundance of Actinobacteria with the low C/N ratio was 11-20\% higher than that of the other treatments, indicating that the difference in porosity caused by particle size started to affect the composting process in the middle stage and became an important influencing factor.

In the maturity phase, Chloroflexi replaced Proteobacteria to become the dominant phylum, with the average abundance increasing from the initial $0.5 \%$ to $30 \%$; Acidobacteria also continued to increase to 1.8 times that in the middle stage; and the abundances of other phyla decreased compared to those in the middle stage, which is because with the degradation of easily degraded organic matter in the early and middle stages and the stable increase in HS, the number of available nutrients decreased, which led to limited bacterial activity with simple organics as the energy source. The abundance of Acidobacteria for the $2 \mathrm{~mm}$ treatments was $41.8 \%$ higher than that for the $5 \mathrm{~mm}$ treatments, and the average abundance of the low-C/N treatments was $18 \%$ higher than that of the other treatments. The differences were not significant for Chloroflexi.

The top 20 orders of composting were characterized to complement and confirm the function of bacterial activity in the GWC (Fig. 5b). The choice of order level was based on the comprehensive classification hierarchy and identification rate to avoid a biased explanation (Shen et al., 2013). At the same time, LDA Effect Size analysis was used to analyze the bacterial community differences between groups to determine the specific major microbial population in groups. The results showed that the specific major bacteria species, which had significant effects (LDA>3) were consistent with the top 20 orders basically. Specifically, Clostridiales, Bacillales, Sphingobacteriaceae, Sphingomonadales and Burkholderiales had the most significant influence at the early stage. Studies have shown that Clostridiales and Bacillales widely occur during composting due to their good degradation ability. In particular, Bacillus is commonly found in lignocellulose composting systems and can rapidly colonize and decompose lignocellulolytic substrates (Tortosa et al., 2017). Sphingobacteriaceae is known for its ability to utilize a variety of $\mathrm{C}$ sources, and it is reduced by the decomposition of carbon resources (Anderson et al., 2011). Therefore, Sphingobacteriaceae also plays an important role in the initial stage of composting. Streptosporangiales, Rhizobiales, Rhodospirillales and Phycisphaerales have a significant impact in the middle stage, and they are all closely related to the $\mathrm{C}$ or $\mathrm{N}$ cycles (Anderson et al., 2011; Badhai et al., 
2015). Rhodospirillales can exhibit thermophilicity. With the decomposition of TOC and TN, the number of these bacteria gradually decreased. Anaerolineales, Blastocatellales, and Chloroflexales become the dominant orders in the late stage. These orders can promote the production of carboxymethyl cellulose, which is involved in the degradation of polysaccharides and lignocellulose compounds (Tortosa et al., 2017; Awasthi et al., 2017b). Anaerolinaceae was the major order in the final composting stage, with the average abundance rising from less than $0.1 \%$ to $18.5 \%$. Studies have shown that these bacteria can digest carbohydrates and peptides and may promote the utilization of carbohydrates or amino acids in dead cells (Yamada et al., 2006), which promotes the polymerization of HS. These results provide insights into the complex microbial activities involved in the composting process, clarify the microbial species that have a significant impact on the composting process, and indicate potential methods that can be used future studies to improve composting quality via the inoculation and cultivation of key bacteria.

\section{Relationships among environmental factors, bacterial communities and HS}

According to $\mathrm{Wu}$ et al. (2017), environmental factors (including physicochemical parameters and precursors) and key bacteria are the main factors that affect the formation of HS. To further clarify the relationship among the three factors and determine the key factors affecting the formation of HS, we performed a correlation analysis on the environmental factors, bacterial communities and HS.

A Pearson correlation analysis was conducted between the environmental factors and humification parameters to study their possible role in the composting process (Fig. 6a) (Dias et al., 2010). The results showed that GI, temperature, polyphenols and polysaccharides had a strong correlation with humification parameters $(r>0.75, p<0.05)$, thus indicating that these were key environmental factors affecting the formation of HS. Polyphenols and carboxyl were significantly negatively correlated with humification parameters, confirming that carboxyl groups and phenyl groups are the main substances that constitute the total acidity (deMelo et al., 2016), promote the formation of HS through structural integration and increase the aromaticity of HS. Polysaccharides were positively related with HS because these substances are formed via the degradation of lignin, cellulose and hemicellulose (Sánchez-Monedero et al., 1999) and can be used as the main carbon and energy sources of bacterial communities. We can infer that polysaccharides are the main energy source of the bacteria in the late stage and can promote the bacterial aggregation effect of small molecules, thus promoting the formation of HS. These results also support the idea that precursors play an important role in HS formation and aromatization. Previous studies also found that temperature and GI are related to bacteria composition. Temperature affects bacteria community succession and composition, and GI, as a maturity index, is associated with the phytotoxicity, which significantly affects bacterial activity. Therefore, temperature and GI can significantly affect the composting process by affecting bacterial communities.

As mentioned above, the top 20 orders were selected to analyze the relationship between the bacterial community and humification parameters and elaborate on the influence of bacterial communities on HS. As shown in the Fig. 6b, Anaerolineales, Chloroflexales, Blastocatellales, Cytophagales, Myxococcales and Nitrosomonadales were positively correlated with HS, HA, HI, HR, PHA and DP and negatively correlated with $\mathrm{FA}$ and $\mathrm{E}_{4} / \mathrm{E}_{6}$, which play positive roles in the composting process. According to the Pearson correlation analysis and previous reports (Tortosa et al., 2017), some genera 
of the aforementioned orders could be used as biomarkers for the thermophilic phase and maturity phase in the composting. Among them, the OTUs of Anaerolineales, Cytophagales and Blastocatellales increased as composting progressed and were significantly correlated with all indices $(\mathrm{r}>0.75, \mathrm{p}<0.05)$. Therefore, Anaerolineales, Cytophagales and Blastocatellales could be considered as important microorganisms affecting the maturity of GWC. Previous studies have confirmed that Anaerolineales and Cytophagaceae can digest proteins, cellulose and other macromolecules (Meng et al., 2018) to produce nitrogen and sugar, which may promote the formation of HS. However, more research is needed to confirm these results.

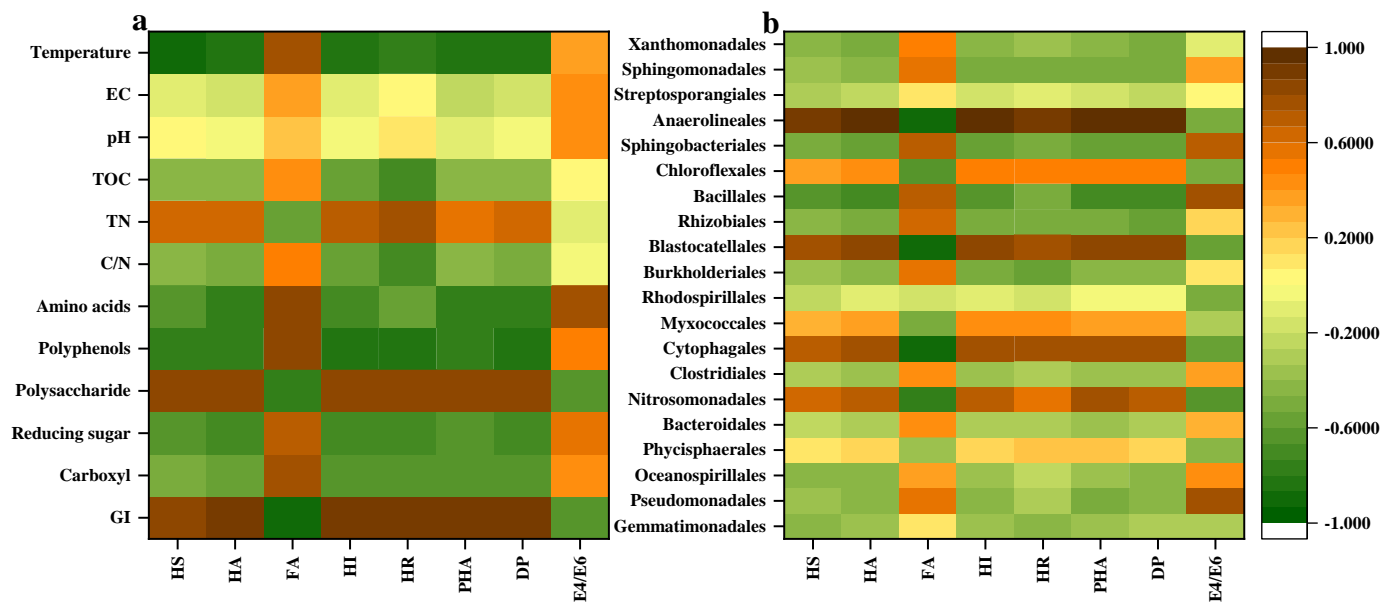

Figure 6. Pearson correlation heatmap of a) environmental factors and humification parameters and b) bacterial communities and humification parameters

According to Wang et al. (2015), environmental factors may be the most manageable factors affecting HS formation. Thus, exploring the influence of environmental factors on bacterial communities is of great important to regulate the production of HS more conveniently. However, the relationship between the two in the GWC remains unknown. Therefore, redundancy analysis (RDA) is performed based on environmental factor parameters and bacterial abundance (Fig. 7). Results of the Monte Carlo test showed that the first and all canonical axes were highly significant $(\mathrm{p}=0.002)$, moreover, eigenvalues showed that the total variation in the bacterial abundance statistically explained by these environmental factors accounting for $97.3 \%$, indicating that these environmental factors may be significant in explaining bacterial community formation.

RDA indicated the relationship between environmental parameters and bacteria communities abundance at the order level. During the early stage of composting, the major bacteria (Sphingobacteriales, Pseudomonadales, Burkholderiales, and Sphingomonadales) were affected by more than one environmental factor, suggesting their growth in an intricate environment. Key bacterial and small molecule precursors (amino acids, reducing sugars, polyphenols and carboxyl) directly involved in the formation of HS were positively correlated. Therefore, the values of these indices could be increased in the initial stage to provide more nutrients and promote the composting process. Moreover, bacteria could also facilitate the generation and polymerization of these functional precursors by decomposing readily degradable cellulose and 
hemicellulose, to produce more HS. As a key environmental factor, temperature had a certain impact on the major bacteria in the early and middle stages, and the positive correlation indicates that it can promote the composting process. However, temperature was negatively correlated with the formation of HS, indicating that the cooling stage after the thermophilic phase was more suitable for the formation of HS. Anaerolineales, Cytophagales, Blastocatellales and Nitrosomonadales were positively correlated with the composting samples in the late stage and the formation of HS, indicating that HS formation mainly occurred in the late stage and these bacteria may be related to the polymerization of substance.

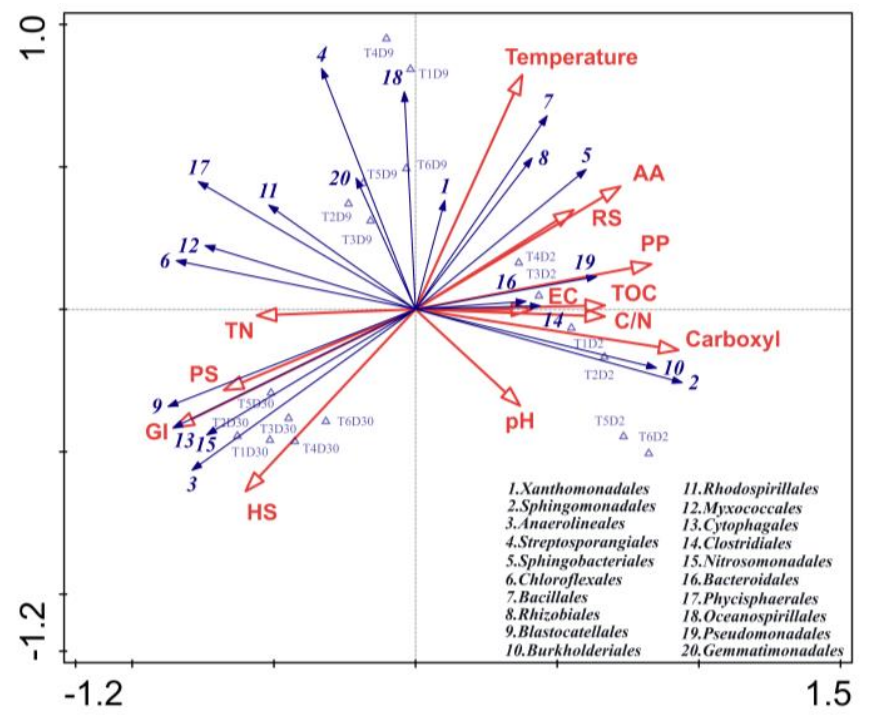

Figure 7. RDA of the correlation between environmental factors, bacterial community and compost samples (AA: amino acids, PP: polyphenols, PS: polysaccharides, RS: reducing sugars)

Anaerolineales, Cytophagales, and Blatocatellales are the key bacteria affecting HS, and they were positively correlated with GI, polysaccharides, and TN, indicating that the high lignin content of GW resulted in very low availability of nutrients in the late stage of composting and suggesting that polysaccharides and TN may be the main sources of energy for bacteria, which confirms our previous inference. The content and composition of nitrogen are considered to influence the bacterial community through the availability of essential elements that affect the bacterial survival (Chodak et al., 2013; Xi et al., 2016). We can increase the activity of key bacteria through the addition of various materials, such as bean dregs, fruit and vegetable waste, in the late stage to promote the formation of HS and effectively control the cost of composting. As an important biological indicator, GI is significantly related to quinone group, compost maturity and phytotoxicity (Tang et al., 2011). Increasing the abundance of these key bacteria could increase the content of terpenoids, thereby improving the phytotoxicity and maturity of composting.

Although we have provided some suggestions for increasing the HS of GWC, the complexity of the composting process necessitates further study of the aspects of operability, cost, and quantitative control so that more precise and effective regulations can be developed to improve the potential applicability of the suggested techniques. 


\section{Conclusions}

In this study, different IPS and $\mathrm{C} / \mathrm{N}$ ratios were used, the dynamic changes in environmental factors and bacterial communities during the GWC were studied, and the correlations between these factors and the formation of HS were revealed. The results showed that the $2 \mathrm{~mm}$ and $\mathrm{C} / \mathrm{N}=23$ treatment could accelerate mineralization, increase fermentation temperature and GI, and generate a high HS content and humification degree. Temperature, GI, polyphenols, and polysaccharides are environmental factors that significantly affect the formation of HS; and Analonineales, Cytophagales, and Blastocatellales are important bacteria that affect the maturity process. In future studies, various spectroscopy techniques (e.g. Fourier transform infrared spectroscopy, nuclear magnetic resonance) can be used to explore the mechanism of HS formation in green waste composting from the perspective of material structure, so as to further improve the humification process and the quality of HS. Meanwhile, regulation methods suitable for different scales of composting should be proposed from the perspectives of cost and operability.

Acknowledgement. The authors are grateful for financial support from National Key R\&D Program of China (2017YFC0504406). Thank American Journal Experts for their linguistic modification of this paper.

\section{REFERENCES}

[1] Anderson, C. R., Condron, L. M., Clough, T. J., Fiers, M., Stewart, A., Hill, R. A., Sherlock, R. R. (2011): Biochar induced soil microbial community change: Implications for biogeochemical cycling of carbon, nitrogen and phosphorus. - Pedobiologia 54: 309320 .

[2] Antunes, L. P., Martins, L. F., Pereira, R. V., Thomas, A. M., Barbosa, D., Lemos, L. N. (2016): Microbial community structure and dynamics in thermophilic composting viewed through metagenomics and metatranscriptomics. - Scientific Reports 6(1): 38915.

[3] Ariesyady, H. D., Ito, T., Okabe, S. (2007): Functional bacterial and archaeal community structures of major trophic groups in a full-scale anaerobic sludge digester. - Water Research 41(7): 1554-1568.

[4] Awasthi, M. K., Pandey, A. K., Khan, J., Bundela, P. S., Wong, J. W. C., Selvam, A. (2014): Evaluation of thermophilic fungal consortium for organic municipal solid waste composting. - Bioresource Technology 168: 214-221.

[5] Awasthi, M. K., Li, J., Kumar, S., Awasthi, S. K., Wang, Q., Chen, H. Y., Wang, M. J., Ren, X. N., Zhang, Z. Q. (2017a): Effects of biochar amendment on bacterial and fungal diversity for co-composting of gelatin industry sludge mixed with organic fraction of municipal solid waste. - Bioresource Technology 246: 214-223.

[6] Awasthi, M. K., Zhang, Z., Wang, Q., Shen, F., Li, R. H., Li, D. S., Ren, X. N., Wang, M. J., Chen, H. Y., Zhao, J. C. (2017b): New insight with the effects of biochar amendment on bacterial diversity as indicators of biomarkers support the thermophilic phase during sewage sludge composting. - Bioresource Technology 238: 589-601.

[7] Badhai, J., Ghosh, T. S., Das, S. K. (2015): Taxonomic and functional characteristics of microbial communities and their correlation with physicochemical properties of four geothermal springs in Odisha. - Frontiers in Microbiology 6: 1166.

[8] Barje, F., El Fels, L., El Hajjouji, H., Amir, S., Winterton, P., Hafidi, M. (2012): Molecular behaviour of humic acid-like substances during co-composting of olive mill 
waste and the organic part of municipal solid waste. - International Biodeterioration \& Biodegradation 74: 17-23.

[9] Chen, W., Liao, X., Wu, Y., Liang, J. B., Mi, J., Huang, J., Zhang, H., Wu, Y., Qiao, Z., Li, X., Wang, Y. (2017): Effects of different types of biochar on methane and ammonia mitigation during layer manure composting. - Waste Management 61: 506-515.

[10] Chodak, M., Golebiewski, M., Morawska-Ploskonka, J., Kuduk, K., Niklinska, M. (2013): Diversity of microorganisms from forest soils differently polluted with heavy metals. - Applied Soil Ecology 64: 7-14.

[11] deMelo, B. A. G., Motta, F. L., Santana, M. H. (2016): Humicacids: Structural properties and multiple functionalities for novel technological developments. - Materials Science and Engineering C 62: 967-974.

[12] Dias, B. O., Silva, C. A., Higashikawa, F. S., Roig, A., Sánchez-Monedero, M. A. (2010): Use of biochar as bulking agent for the composting of poultry manure: effect on organic matter degradation and humification. - Bioresource Technology 101: 1239-1246.

[13] Dzulkurnain, Z., Hassan, M. A., Zakaria, M. R., Wahab, P. E. M., Hasan, M. Y., Shirai, Y. (2017): Co-composting of municipal sewage sludge and landscaping waste: a pilot scale study. - Waste and Biomass Valorization 8: 1-11.

[14] Guo, X. X., Liu, H. T., Wu, S. B. (2019): Humic substances developed during organic waste composting: Formation mechanisms, structural properties, and agronomic functions. - Science of The Total Environment 662: 501-510.

[15] Hanc, A., Dreslova, M. (2016): Effect of composting and vermicomposting on properties of particle size fractions. - Bioresource Technology 217: 186-189.

[16] Huang, G. F., Wong, J. W. C., Wu, Q. T., Nagar, B. B. (2004): Effect of C/N on composting of pig manure with sawdust. - Waste Management 24(8): 805-813.

[17] Khalil, A., Domeizel, M., Prudent, P. (2008): Monitoring of green waste composting process based on redox potential. - Bioresource Technology 99(14): 6037-6045.

[18] Kulikowska, D., Klimiuk, E. (2011): Organic matter transformations and kinetics during sewage sludge composting in a two-stage system. - Bioresource Technology 102(23): 10951-10958.

[19] Li, R., Wang, J. J., Zhang, Z., Shen, F., Zhang, G., Qin, R., Li, X., Xiao, R. (2012): Nutrient transformations during composting of pig manure with bentonite. - Bioresource Technology 121: 362-368.

[20] Li, Y., Li, W., Wu, C., Wang, K. (2013): New insights into the interactions between carbon dioxide and ammonia emissions during sewage sludge composting. - Bioresource Technology 136: 385-393.

[21] Li, X., Hu, Q., Jiang, S., Li, F., Lin, J., Han, L., Hong, Y., Lu, W., Gao, Y., Chen, D. (2015): Flos Chrysanthemi Indici protects against hydroxyl-induced damages to DNA a MSCs via antioxidant mechanism. - Journal of Saudi Chemical Society 19(4): 454-460.

[22] Liu, L., Wang, S., Guo, X., Zhao, T., Zhang, B. (2018): Succession and diversity of microorganisms and their association with physicochemical properties during green waste thermophilic composting. - Waste Management 73: 101-112.

[23] Lobna, B., Guergueb, Z., Chaieb, M., Mekki, A. (2019): Co-composting of Olive Industry Wastes with Poultry Manure and Evaluation of the Obtained Compost Maturity.

- Waste and Biomass Valorization. DOI: 10.1007/s12649-019-00901-9.

[24] López-González, J. A., Suárez-Estrella, F., Vargas-García, M. C., López, M. J., Jurado, M. M., Moreno, J. (2015): Dynamics of bacterial microbiota during lignocellulosic waste composting: studies upon its structure, functionality and biodiversity. - Bioresource Technology 175: 406-416.

[25] Meng, X., Liu, B., Xi, C., Luo, X., Yuan, X., Wang, X., Zhu, W., Wang, H., Cui, Z. (2018): Effect of pig manure on the chemical composition and microbial diversity during co-composting with spent mushroom substrate and rice husks. - Bioresource Technology 251: $22-30$. 
[26] Petric, I., Helić, A., Avdić, E. A. (2012): Evolution of process parameters and determination of kinetics for co-composting of organic fraction of municipal solid waste with poultry manure. - Bioresource Technology 117: 107-116.

[27] Sánchez-Monedero, M. A., Roig, A., Cegarra, J., Bernal, M. P. (1999): Relationships between water-soluble carbohydrate and phenol fractions and the humification indices of different organic wastes during composting. - Bioresource Technology 70: 193-201.

[28] Shen, P., Zhang, J., Zhang, J., Jiang, C., Tang, X., Li, J., Zhang, M., Wu, B. (2013): Changes in microbial community structure in two anaerobic systems to treat bagasse spraying wastewater with and without addition of molasses alcohol wastewater. Bioresource Technology 131: 333-340.

[29] Silva, M. E. F., de Lemos, L. T., Nunes, O. C., Cunha-Queda, A. C. (2014): Influence of the composition of the initial mixtures on the chemical composition, physicochemical properties and humic-like substances content of composts. - Waste Management 34: 2127.

[30] Song, C., Li, M., Jia, X., Wei, Z., Zhao, Y., Xi, B., Zhu, C., Liu, D. (2014): Comparison of bacterial community structure and dynamics during the thermophilic composting of different types of solid wastes: anaerobic digestion residue, pig manure and chicken manure. - Microbial Biotechnology 7: 424-433.

[31] Taha, M., Foda, M., Shahsavari, E., Aburto-Medina, A., Adetutu, E., Ball, A. (2016): Commercial feasibility of lignocellulose biodegradation: possibilities and challenges. Current Opinion in Biotechnology 38: 190-197.

[32] Tang, J. C., Wang, M., Zhou, Q. X., Nagata, S. (2011): Improved composting of Undaria pinnatifida seaweed by inoculation with Halomonas and Gracilibacillus sp. isolated from marine environments. - Bioresource Technology 102: 2925-2930.

[33] Tortosa, G., Castellano-Hinojosa, A., Correa-Galeote, D., Bedmar, E. J. (2017): Evolution of bacterial diversity during two-phase olive mill waste ("alperujo") composting by 16S rRNA gene pyrosequencing. - Bioresource Technology 224: 101111.

[34] Wang, X., Cui, H., Shi, J., Zhao, X., Zhao, Y., Wei, Z. (2015): Relationship between bacterial diversity and environmental parameters during composting of different raw materials. - Bioresource Technology 198: 395-402.

[35] Wang, S. P., Zhong, X. Z., Wang, T. T., Sun, Z. Y., Tang, Y. Q., Kida, K. (2017): Aerobic composting of distilled grain waste eluted from a Chinese spiritmaking process: the effects of initial $\mathrm{pH}$ adjustment. - Bioresource Technology 245: 778-785.

[36] Wei, H., Wang, L., Hassan, M., Xie, B. (2018): Succession of the functional microbial communities and the metabolic functions in maize straw composting process. Bioresource Technology 256: 333-341.

[37] Wu, J., Zhao, Y., Zhao, W., Yang, T., Zhang, X., Xie, X., Cui, H., Wei, Z. (2017): Effect of precursors combined with bacteria communities on the formation of humic substances during different materials composting. - Bioresource Technology 226: 191-199.

[38] Xi, B. D., Zhao, X. Y., He, X. S., Huang, C. H., Tan, W. B., Gao, R. T., Zhang, H., Li, D. (2016): Successions and diversity of humic-reducing microorganisms and their association with physical-chemical parameters during composting. - Bioresource Technology 219: 204-211.

[39] Yamada, T., Sekiguchi, Y., Hanada, S., Imachi, H., Ohashi, A., Harada, H., Kamagata, Y. (2006): Anaerolinea thermolimosa sp nov., Levilinea saccharolytica gen. nov., sp nov and Leptolinea tardivitalis gen. nov., so. nov., novel filamentous anaerobes, and description of the new classes anaerolineae classis nov and Caldilineae classis nov in the bacterial phylum Chloroflexi. - International Journal of Systematic and Evolutionary Microbiology 56: 1331-1340.

[40] Zhang, L., Sun, X. Y. (2014): Effects of rhamnolipid and initial compost particle size on the two-stage composting of green waste. - Bioresource Technology 163: 112-122. 


$$
-62-
$$

[41] Zhang, L. L., Zhang, H. Q., Wang, Z. H., Chen, G. J., Wang, L. S. (2016): Dynamic changes of the dominant functioning microbial community in the compost of a 90-m3 aerobic solid state fermentor revealed by integrated meta-omics. - Bioresource Technology 203: 1-10.

[42] Zhang, L., Sun, X. Y. (2018): Effects of bean dregs and crab shell powder additives on the composting of green waste. - Bioresource Technology 260: 283-293.

[43] Zhang, Z. C., Zhao, Y., Wang, R. X., Lu, Q., Wu, J. Q., Zhang, D. Y., Nie, Z. F., Wei, Z. M. (2018): Effect of the addition of exogenous precursors on humic substance formation during composting. - Waste Management 79: 462-471.

[44] Zhang, L., Sun, X. Y. (2019): The use of coal fly ash and vinegar residue as additives in the two-stage composting of green waste. - Environmental science and pollution research. In Press.

[45] Zhang, W. M., Yu, C. X., Wang, X. J., Hai, L. (2020): Increased abundance of nitrogen transforming bacteria by higher $\mathrm{C} / \mathrm{N}$ ratio reduces the total losses of $\mathrm{N}$ and $\mathrm{C}$ in chicken manure and corn stover mix composting. - Bioresource Technology 297: 122410.

[46] Zhao, X., He, X., Xi, B., Gao, R., Tan, W., Zhang, H., Li, D. (2016): The evolution of water extractable organic matter and its association with microbial community dynamics during municipal solid waste composting. - Waste Management 56: 79-87.

[47] Zhou, Y., Selvam, A., Wong, J. W. C. (2014): Evaluation of humic substances during cocomposting of food waste, sawdust and Chinese medicinal herbal residues. - Bioresource Technology 168: 229-234.

[48] Zhou, G. X., Xu, X. F., Qiu, X. W., Zhang, J. B. (2019): Biochar influences the succession of microbial communities and the metabolic functions during rice straw composting with pig manure. - Bioresource Technology 272: 10-18.

[49] Zhu, N. (2007): Effect of low initial C/N ratio on aerobic composting of swine manure with rice straw. - Bioresource Technology 98: 9-13.

[50] Zucconi, F., Pera, A., Forte, M., De Bertoldi, M. (1981): Evaluating toxicity of immature compost. - Biocycle 22: 54-57. 